\title{
Proposed Experiment for Testing Quantum Contextuality with Neutrons
}

\author{
Adán Cabelld* \\ Departamento de Física Aplicada II, Universidad de Sevilla, E-41012 Sevilla, Spain \\ Stefan Filipp † $^{-1}$ and Helmut Rauch \\ Atominstitut der Österreichischen Universitäten, Stadionallee 2, A-1020 Vienna, Austria \\ Yuji Hasegawa \\ Atominstitut der Österreichischen Universitäten, Stadionallee 2, A-1020 Vienna, Austria and \\ PRESTO, Japan Science and Technology Agency (JST), Kawaguchi, Saitama 332-0012, Japan
}

(Dated: November 3, 2018)

\begin{abstract}
We show that an experimental demonstration of quantum contextuality using 2 degrees of freedom of single neutrons based on a violation of an inequality derived from the Peres-Mermin proof of the Kochen-Specker theorem would be more conclusive than those obtained from previous experiments involving pairs of ions [M. A. Rowe et al., Nature (London) 409, 791 (2001)] and single neutrons [Y. Hasegawa et al., Nature (London) 425, 45 (2003)] based on violations of Clauser-Horne-ShimonyHolt-like inequalities.

PACS numbers: 03.65.Ud, 03.75.Dg, 07.60.Ly, 42.50.Xa
\end{abstract}

Bell inequalities are constraints imposed by local hidden-variable theories (LHVTs) on the values of some specific linear combinations of the averages of the results of spacelike separated experiments on distant systems. From a conclusive experimental violation of a Bell inequality we would learn that no local hidden-variable theory can describe the world we see. This "quantum nonlocality" (i.e., the fact that quantum mechanics [QM] cannot be reproduced by LHVTs [1]) is supposed to be universal, in the sense that we should be able to detect it by using any composite quantum system (photons, electrons, neutrons, atoms, molecules). However, in practice, so far we do not have a loophole-free experimental violation of a Bell inequality, and, specifically we do not have any Bell inequality test involving spacelike separated experiments with any quantum system apart of photons [2, 3, 4]. Therefore, an interesting question is what can we learn about the possibility of reproducing experimental observations with hidden-variable theories from experiments performed on massive nonspacelike separated quantum systems such as neutrons and atoms.

LHVTs are a subset of a larger class of hidden-variable theories known as noncontextual hidden-variable theories (NCHVTs). NCHVTs are defined as those in which the result of a measurement of an observable is assumed to be predetermined and not affected by a (previous or simultaneous) suitable measurement of any other compatible (i.e., comeasurable) observable.

In this sense, some recent experiments testing Bell inequalities on pairs of two-level ${ }^{9} \mathrm{Be}^{+}$ions separated by a distance of $3 \mu \mathrm{m}$ [5] and two two-level degrees of freedom (spatial and spin components) of single neutrons [6] cannot be considered tests of LHVTs, but only tests of NCHVTs (see, for instance [7, 8]). Both mentioned experiments have in common the fact that they test the
Clauser-Horne-Shimony-Holt Bell inequality [9],

$$
\left|\left\langle A_{0} B_{0}\right\rangle+\left\langle A_{0} B_{1}\right\rangle+\left\langle A_{1} B_{0}\right\rangle-\left\langle A_{1} B_{1}\right\rangle\right| \leq 2 .
$$

Common to both experiments is also the fact that they have very good overall detection efficiency, both around 99\% (specially when compared with that of Bell tests with photons which is around 5\% [4]). This very good detection efficiency would allow us to avoid the fair sampling assumption (i.e., that the detected particles are a representative sample of all emitted ones) needed to reach any conclusion in photon experiments of LHVTs [2, 3, 4] However, common to both experiments is also the fact that, due to experimental imperfections (imperfect state creation, manipulations, and detection), the final experimental results $(2.25 \pm 0.03$ [5] and $2.051 \pm 0.019$ [6]) are indeed closer to the hidden-variable bound, 2 , than to the maximal violation predicted by $\mathrm{QM}, 2 \sqrt{2} \approx 2.82$. Therefore, an interesting question is whether we can design a better "quantum contextuality" experiment (i.e., showing us that no NCHVT can describe the world we see) for these systems. By a better experiment we mean one in which the observed violation expected from the hiddenvariable bound would be significantly higher than those obtained in these previous experiments.

One possible answer comes from the fact that the observation that QM cannot be reproduced by NCHVTs has its own history starting from Kochen-Specker theorem [10, 11, 12] and has developed its own proofs apart from those of Bell's theorem. These proofs are specifically designed to stress the difference between QM and NCHVTs. In this Letter, we show that it is possible to demonstrate quantum contextuality using massive quantum systems, and specifically 2 degrees of freedom of single neutrons following the steps of a state-dependent multiplicative proof of the KS theorem [13] proposed by 
Peres [14] and Mermin (who extended Peres' proof into a state-independent proof [15, 16], which can be converted into a standard proof of the KS theorem [17], which indeed contains the simplest possible [18] standard proof of the KS theorem [19]).

The Peres-Mermin proof, suitably adapted, for instance, to the case of the two two-level degrees of freedom (spatial and spin components) of single neutrons [6] is as follows. Consider a system prepared in the state

$$
|\Psi\rangle=\frac{1}{\sqrt{2}}(|\downarrow\rangle \otimes|I\rangle-|\uparrow\rangle \otimes|I I\rangle),
$$

where $|\uparrow\rangle$ and $|\downarrow\rangle$ denote the up-spin and down-spin states, and $|I\rangle$ and $|I I\rangle$ denote the two beam paths in an interferometer. $s$ stands for spin and $p$ for path. Let us suppose that the six observables $\sigma_{x}^{s}, \sigma_{y}^{s}, \sigma_{x}^{p}, \sigma_{y}^{p}, \sigma_{x}^{s} \sigma_{y}^{p}$, and $\sigma_{y}^{s} \sigma_{x}^{p}$ have predefined values $v\left(\sigma_{x}^{s}\right), v\left(\sigma_{y}^{s}\right), v\left(\sigma_{x}^{p}\right), v\left(\sigma_{y}^{p}\right)$, $v\left(\sigma_{x}^{s} \sigma_{y}^{p}\right)$, and $v\left(\sigma_{y}^{s} \sigma_{x}^{p}\right)$, respectively. For the state $|\Psi\rangle$, the predictions of QM used in the proof are the following five:

$$
\begin{aligned}
v\left(\sigma_{x}^{s}\right) v\left(\sigma_{x}^{p}\right) & =-1, \\
v\left(\sigma_{y}^{s}\right) v\left(\sigma_{y}^{p}\right) & =-1, \\
v\left(\sigma_{x}^{s} \sigma_{y}^{p}\right) v\left(\sigma_{x}^{s}\right) v\left(\sigma_{y}^{p}\right) & =1, \\
v\left(\sigma_{y}^{s} \sigma_{x}^{p}\right) v\left(\sigma_{y}^{s}\right) v\left(\sigma_{x}^{p}\right) & =1, \\
v\left(\sigma_{x}^{s} \sigma_{y}^{p}\right) v\left(\sigma_{y}^{s} \sigma_{x}^{p}\right) & =-1 .
\end{aligned}
$$

Equations (3a) and (3b) follow from the anticorrelations designed into the state $|\Psi\rangle$, Eqs. (3c) and (3d) follow from the fact that the values assigned to mutually commuting operators whose product is the identity must obey the same relation satisfied by the operators themselves, and Eq. (3e) follows from the fact that $\left(\sigma_{x}^{s} \otimes \sigma_{y}^{p}\right)\left(\sigma_{y}^{s} \otimes \sigma_{x}^{p}\right)=\sigma_{z}^{s} \otimes \sigma_{z}^{p}$ and the anticorrelations of the state $|\Psi\rangle$.

To show that it is impossible to ascribe predefined values -1 or 1 to each and every of these six observables, it is enough to multiply both sides of Eqs. (3a)-(3e): Each observable appears twice; therefore, we have 1 as the product of the left-hand sides. However, we have -1 as the product of the right-hand sides 14, 15, 16].

An ideal experiment for discarding NCHVTs would be simply to confirm each and every of these five predictions of QM. An important point is that each equation corresponds to a different experimental context. Therefore, the experiment requires to perform five different types of experiments. A fundamental point is the measurement apparatus used for measuring, e.g., $\sigma_{x}^{s} \sigma_{y}^{p}$ must be the same in the experimental context corresponding to Eq. (3c) and in that corresponding to Eq. (3e).

Then, since the perfect correlations (or anticorrelations) on which the proof is based are not obtained in real experiments, one should derive a Bell-like inequality. Some inequalities for quantum contextuality have been proposed [20, 21, 22, 23]. The one introduced here has two advantages: it is the direct translation of the PeresMermin proof into an experimentally testable inequality, and provides a significant contrast between the prediction of QM and the hidden-variable bound.

The relevant properties of the Peres-Mermin proof come from Eqs. (3a)-(3e). Then, a reasonable choice is to investigate the inequality obtained by the linear combination of the averages of the results obtained in the five experimental contexts, with the same coefficients $(-1$ or 1) appearing in the quantum predictions [i.e., in Eqs. (3a) - (3e)]. For this inequality, it can be proven (for instance, by writing a simple computer program) that, in any NCHVT,

$$
\begin{aligned}
-\left\langle\sigma_{x}^{s}\right. & \left.\cdot \sigma_{x}^{p}\right\rangle-\left\langle\sigma_{y}^{s} \cdot \sigma_{y}^{p}\right\rangle+\left\langle\sigma_{x}^{s} \sigma_{y}^{p} \cdot \sigma_{x}^{s} \cdot \sigma_{y}^{p}\right\rangle \\
& +\left\langle\sigma_{y}^{s} \sigma_{x}^{p} \cdot \sigma_{y}^{s} \cdot \sigma_{x}^{p}\right\rangle-\left\langle\sigma_{x}^{s} \sigma_{y}^{p} \cdot \sigma_{y}^{s} \sigma_{x}^{p}\right\rangle \leq 3
\end{aligned}
$$

while the prediction of QM is just 5 , as can be seen from the predictions of QM leading to Eqs. (3a - (3e). This is the inequality that one should test. Such a test requires five different types of experiments and requires measuring any observable using the same apparatus independently of the context in which it appears.

However, it is interesting to note that the five experiments in Eqs. (3a)-3e play different roles in the proof. Note the difference between Eqs. (3c) and (3d) and the other three equations (3a), (3b), and (3e). While Eqs. (3c) and (3d) should hold for any preparation (i.e., are state-independent predictions of both QM and NCHVTs), Eqs. (3a), (3b), and (3e) are predictions specific for a particular quantum state (2).

Since Eqs. (3c) and (3d) hold both in QM and in NCHVTs and do not depend on any particular preparation of the state, we do not really need to test them. This observation was first made in [24] and was used in several Bell-like inequalities for NCHVTs. Therefore, it is enough to test the following Bell-like inequality:

$$
-\left\langle\sigma_{x}^{s} \cdot \sigma_{x}^{p}\right\rangle-\left\langle\sigma_{y}^{s} \cdot \sigma_{y}^{p}\right\rangle-\left\langle\sigma_{x}^{s} \sigma_{y}^{p} \cdot \sigma_{y}^{s} \sigma_{x}^{p}\right\rangle \leq 1
$$

However, and this is an important point missed in previous discussions, an experiment of this type cannot be considered a test of quantum contextuality unless we also describe how, at least in principle, the two predictions not considered in the Bell-like inequality (5) can be tested using the same measuring apparatus used to measure the observables in the inequality (5). This means that, for instance, the measuring apparatus used for measuring $\sigma_{x}^{s} \sigma_{y}^{p}$ must be the same regardless of whether it is measured together with $\sigma_{x}^{s}$ and $\sigma_{y}^{p}$, or together with $\sigma_{y}^{s} \sigma_{x}^{p}$. Without such a prescription, an experiment to test the inequality (5) cannot be considered a contextuality experiment, since the six observables involved are just tested in one context and no description of how they can be tested in a different context is provided.

Another motivation for this Letter comes from the fact that a previous experiment on quantum contextuality with single neutrons 25] does not satisfy the above 
requirements. Specifically, in 25] the measurement of $\sigma_{x}^{s} \sigma_{y}^{p} \cdot \sigma_{y}^{s} \sigma_{x}^{p}$ was accomplished by measuring $\sigma_{z}^{s} \sigma_{z}^{p}$. This method is unsuitable, because a measurement of $\sigma_{z}^{s} \sigma_{z}^{p}$ gives only the value of the product $\sigma_{x}^{s} \sigma_{y}^{p} \cdot \sigma_{y}^{s} \sigma_{x}^{p}$, and because the observable $\sigma_{z}^{s} \sigma_{z}^{p}$ is not compatible with the observables $\sigma_{x}^{s}$ and $\sigma_{y}^{p}$, and therefore $\sigma_{z}^{s} \sigma_{z}^{p}$ cannot be used (even potentially) to perform the subsequent required measurements (e.g., $\sigma_{x}^{s} \sigma_{y}^{p}$ together with $\sigma_{x}^{s}$ and $\sigma_{y}^{p}$ ).

Measuring $\sigma_{x}^{s} \sigma_{y}^{p}$ and $\sigma_{y}^{s} \sigma_{x}^{p}$, the two observables in Eq. (3e), simultaneously is typically not a problem (see, e.g., [26, 27]). The challenge is to prove that the apparatuses used for measuring one of them can be combined (potentially) with the subsequent measurements required in Eqs. (3c) and (3d) (e.g., to show that the apparatus for measuring $\sigma_{x}^{s} \sigma_{y}^{p}$ can be combined with a subsequent measurement of $\sigma_{x}^{s}$ and $\sigma_{y}^{p}$ ).

In the following we will describe how to test the inequality (5) using two two-level degrees of freedom (spatial and spin components) of single neutrons, and satisfying all the above mentioned requirements for a proper NCHVT interference experiment. (General descriptions of neutron interferometer experiments are summarized in the literature [28].) A schematic drawing, together with diagrams of the experiments is depicted in Fig. 1) The first half of the interferometer is used for a (Bell) state preparation and the second half is used for the measurement of the path observable, e.g., $\sigma_{x}^{p}$. In addition to auxiliary phase shifters, a pair of spin rotators (dc-coils), which enables arbitrary relative phase and spinor manipulation, is inserted in both parts. After the path measurement, the spinor component, e.g., $\sigma_{x}^{s}$, is measured by the use of a conventional spin analysis system, namely, a spin analyzer accompanied by a spin rotator. Combinations of these path and spin measurements will accomplish the measurements of $\sigma_{x}^{s} \cdot \sigma_{x}^{p}$ and $\sigma_{y}^{s} \cdot \sigma_{y}^{p}$ [illustrated in diagram (i)]. When a spin rotator in one of the beams in the interferometer is tuned to a spin-flip operation and a suitable spin analysis is activated, one can perform the measurement of $\sigma_{x}^{s} \sigma_{y}^{p} \cdot \sigma_{y}^{s} \sigma_{x}^{p}[26,27]$ [cf. diagram (ii)]. The four outcomes correspond to a full Bell-state analysis.

Since it is essential to state a prescription of how one can realize the measurements corresponding to Eqs. (3c) and (3d) with the same apparatus as that corresponding to Eq. (3e), we describe a possible setup for a neutron interferometer experiment. Our proposal consists of using exactly the same apparatus used in case (ii) for measuring $\sigma_{y}^{s} \sigma_{x}^{p} \cdot \sigma_{x}^{s} \sigma_{y}^{p}$, followed by a path and spin measurements after going through a "state-mixer" [shown in case (iii)]. In practice, the "state-mixer" is a beam splitter plus an unitary state rotation. This device forms a quantum eraser [29], which eliminates the information about the results of the noncommuting observable, i.e., $\sigma_{x}^{s} \sigma_{y}^{p}$ in case one would want to measure $\sigma_{y}^{s} \sigma_{x}^{p} \cdot \sigma_{y}^{s} \cdot \sigma_{x}^{p}$ and vice versa: this, in turn, enables measurement of other comeasurable observables. The function of this component is given by a
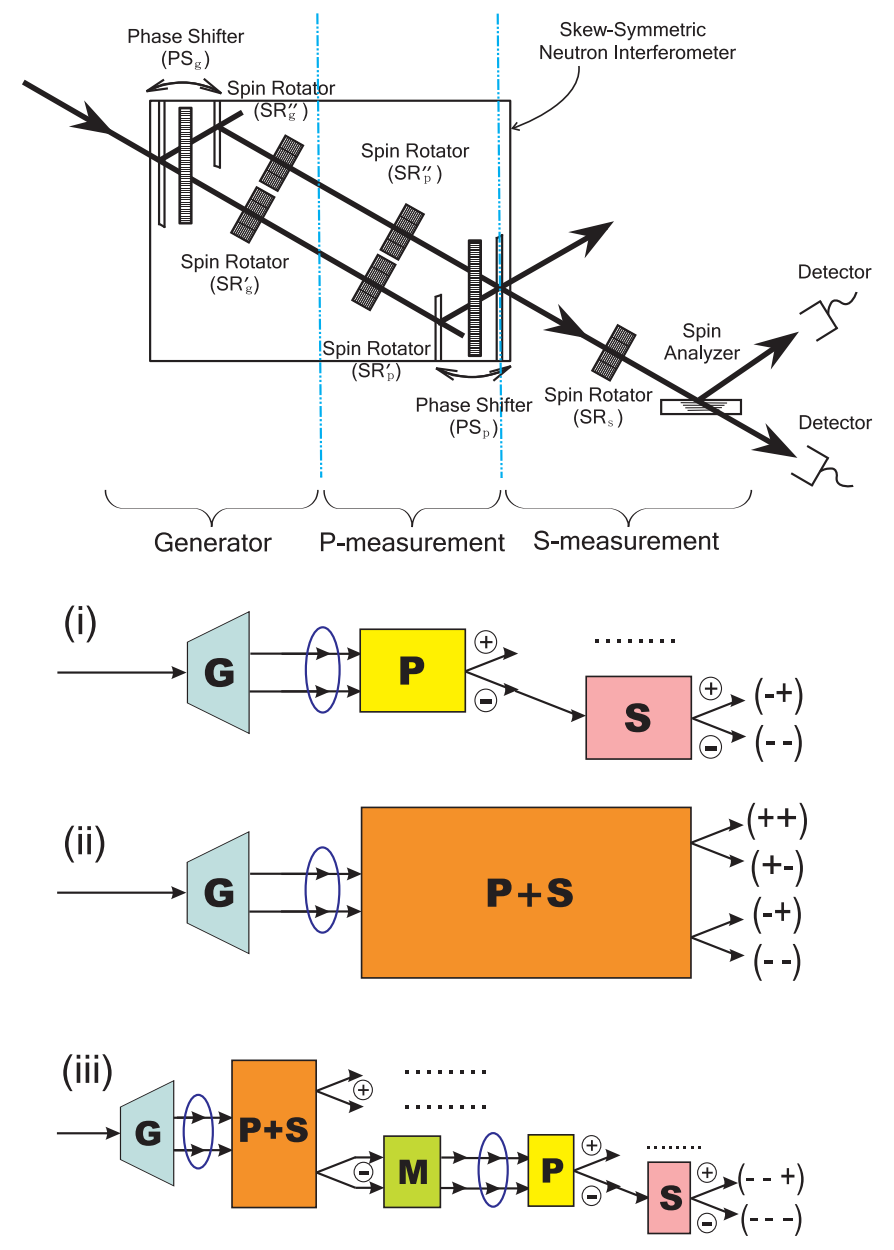

FIG. 1: Above: A proposed experimental setup with a neutron interferometer. The interferometer has two functions: the first half as a state generator, and the second half as a path measurement apparatus. In both parts, a phase shifter (PS) as well as a pair of spin rotators (SR) which enable relative phase and spinor manipulations are inserted. The outgoing beam suffers a spin measurement. Below: Three diagrams for the different measurements required to test the inequality (4). (i) For measurements of $\sigma_{x}^{s} \cdot \sigma_{x}^{p}$ and $\sigma_{y}^{s} \cdot \sigma_{y}^{p}$ : After going through a state generator $(G)$, a state suffers a path measurement $(P)$ followed by a spin measurement $(S)$. Consequently, each outgoing beam gives the results of the two measurements. (ii) For measurements of $\sigma_{y}^{s} \sigma_{x}^{p} \cdot \sigma_{x}^{s} \sigma_{y}^{p}$ : By tuning one of the spin rotators to a spin-flip operation in the path measurement part, the second half of the interferometer together with a spin analyzer $(\mathrm{P}+\mathrm{S})$ work as a Bell-state discriminator: four outgoing beams are assigned to the four possible results of the measurements. (iii) For measurements of $\sigma_{y}^{s} \sigma_{x}^{p} \cdot \sigma_{y}^{s} \cdot \sigma_{x}^{p}$ and $\sigma_{x}^{s} \sigma_{y}^{p} \cdot \sigma_{x}^{s} \cdot \sigma_{y}^{p}$ : After the apparatus $P+S$, a state mixer $(M)$ eliminates the former information about the result of either observable, and is followed by a path and a spin measurement.

projection operator to a state, e.g., $\left|\Psi^{\prime}\right\rangle=\left|\Psi_{1}\right\rangle+\left|\Psi_{2}\right\rangle$, where $\left|\Psi_{1,2}\right\rangle$ are eigenstates of the joint measurement of $\sigma_{x}^{s} \sigma_{y}^{p}$ and $\sigma_{y}^{s} \sigma_{x}^{p}$. It is worth noting here that the device $P+S$ together with the mixer $M$ practically works as a 
$\sigma_{x}^{s} \sigma_{y}^{p}$ or $\sigma_{y}^{s} \sigma_{x}^{p}$ measurement apparatus. This can be tested easily in a separate experiment. As a whole, scheme (iii) can be viewed as the $\sigma_{x}^{s} \sigma_{y}^{p}$ (or $\sigma_{y}^{s} \sigma_{x}^{p}$ ) measurement followed by the $\sigma_{x}^{s}$ and $\sigma_{y}^{p}$ (or $\sigma_{y}^{s}$ and $\sigma_{x}^{p}$ ) measurements. Moreover, the combined measurement scheme (ii) can, in principle, be replaced by these two $\sigma_{x}^{s} \sigma_{y}^{p}$ and $\sigma_{y}^{s} \sigma_{x}^{p}$ measurements in series.

Using data of previous experiments [25] for the first two averages in (5) and estimating the result for the third average from previous experiments on Bell-state discrimination, we estimate that we will find an experimental value above 2.1 (vs. a bound of 1 for NCHVTs), clearly proving quantum contextuality as nowhere else before. The expected value would provide an even clearer quantum contextuality than a recent Mermin-like experiment involving 3 degrees of freedom 30].

Summing up, the contributions of this Letter are the following: We have pointed out that there are better tools to test NCHVTs than the CHSH Bell inequality used in recent experiments with pairs of ions or single photons. A particularly well suited tool is the Peres-Mermin proof [14, 15, 16]. We have described how to implement all the steps required for the Peres-Mermin proof using 2 degrees of freedom of single neutrons. Specifically, we have provided an explicit description of a procedure for measuring observables like $\sigma_{x}^{s} \sigma_{y}^{p}$, which can be followed by a subsequent measurement of the compatible observable $\sigma_{y}^{s} \sigma_{x}^{p}$ or by a subsequent measurement of the compatible observables $\sigma_{x}^{s}$ and $\sigma_{y}^{p}$. In addition, we have derived an inequality which contains the essence of the Peres-Mermin proof, and can be applied to real experiments. All these pieces together result in a proposal of an experiment to test NCHVTs with single neutrons which would presumably improve the results obtained in previous tests with massive quantum systems.

The authors thank K. Svozil for discussions. This research has been supported partly by the Austrian Science Foundation (FWF), Projects No. F1513, No. P18943N20, and No. P17803-N02. A.C. acknowledges support from the Spanish MEC Project No. FIS200507689, and the Junta de Andalucía Excellence Project No. P06-FQM-02243. Y.H. acknowledges support from the Japanese Science and Technology Agency (JST).

* Electronic address: adan@us.es

† Electronic address: sfilipp@ati.ac.at

¥ Electronic address: rauch@ati.ac.at
§ Electronic address: hasegawa@ati.ac.at

[1] J.S. Bell, Physics (Long Island City, NY) 1, 195 (1964).

[2] A. Aspect, J. Dalibard, and G. Roger, Phys. Rev. Lett. 49, 1804 (1982).

[3] W. Tittel, J. Brendel, H. Zbinden, and N. Gisin, Phys. Rev. Lett. 81, 3563 (1998).

[4] G. Weihs, T. Jennewein, C. Simon, H. Weinfurter, and A. Zeilinger, Phys. Rev. Lett. 81, 5039 (1998).

[5] M.A. Rowe, D. Kielpinski, V. Meyer, C.A. Sackett, W.M. Itano, C. Monroe, and D.J. Wineland, Nature (London) 409, 791 (2001).

[6] Y. Hasegawa, R. Loidl, G. Badurek, M. Baron, and H. Rauch, Nature (London) 425, 45 (2003).

[7] L. Vaidman, Phys. Lett. A 286, 241 (2001).

[8] E. Santos, Stud. Hist. Philos. Mod. Phys. 36, 544 (2005).

[9] J.F. Clauser, M.A. Horne, A. Shimony, and R.A. Holt, Phys. Rev. Lett. 23, 880 (1969).

[10] E.P. Specker, Dialectica 14, 239 (1960).

[11] J.S. Bell, Rev. Mod. Phys. 38, 447 (1966).

[12] S. Kochen and E.P. Specker, J. Math. Mech. 17, 59 (1967).

[13] For a classification of the proofs of the KS theorem see A. Cabello and G. García-Alcaine, J. Phys. A 29, 1025 (1996).

[14] A. Peres, Phys. Lett. A 151, 107 (1990).

[15] N.D. Mermin, Phys. Rev. Lett. 65, 3373 (1990).

[16] N.D. Mermin, Rev. Mod. Phys. 65, 803 (1993).

[17] A. Peres, J. Phys. A 24, L175 (1991).

[18] A. Cabello, J.R. Portillo, and G. Potel (unpublished).

[19] A. Cabello, J.M. Estebaranz, and G. García-Alcaine, Phys. Lett. A 212, 183 (1996).

[20] S. Basu, S. Bandyopadhyay, G. Kar, and D. Home, Phys. Lett. A 279, 281 (2001).

[21] C. Simon, Č. Brukner, and A. Zeilinger, Phys. Rev. Lett. 86, 4427 (2001).

[22] J.-A. Larsson, Europhys. Lett. 58, 799 (2002).

[23] J.L. Cereceda, J. Opt. B 4, 87 (2002).

[24] A. Cabello and G. García-Alcaine, Phys. Rev. Lett. 80, 1797 (1998).

[25] Y. Hasegawa, R. Loidl, G. Badurek, M. Baron, and H. Rauch, Phys. Rev. Lett. 97, 230401 (2006).

[26] C. Cinelli, M. Barbieri, R. Perris, P. Mataloni, and F. De Martini, Phys. Rev. Lett. 95, 240405 (2005).

[27] T. Yang, Q. Zhang, J. Zhang, J. Yin, Z. Zhao, M. Żukowski, Z.-B. Chen, and J.-W. Pan, Phys. Rev. Lett. 95, 240406 (2005).

[28] H. Rauch and S.A. Werner, Neutron Interferometry (Clarendon, Oxford, 2000).

[29] M.O. Scully, B.-G. Englert, and H. Walther, Nature (London) 351, 111 (1991).

[30] R. Loidl, J. Klepp, S. Sponar, S. Filipp, G. Badurek, H. Rauch, and Y. Hasegawa, Austrian Physical Society Meeting, Krems, Austria, 24-28 September 2007 (unpublished). In this reference an experimental value of 2.56 is obtained, whereas the bound for NCHVTs is 2 . 